\title{
Análise histológica e histoquímica das fibras dos músculos reto do abdome e intercostal paraesternal de ratos submetidos ao exercício da natação
}

\author{
Paulo César Bálade Saad, Alcides Guimarães, Vitalino Dal Pai e Lúcio Benedicto Kroll \\ Universidade do Oeste Paulista (Unoeste), Presidente Prudente, São Paulo, Brasil
}

\section{RESUMO}

O objetivo deste trabalho foi o de analisar, através de técnicas histológicas e reações histoquímicas, se o exercício induziria alterações nas fibras musculares dos músculos reto do abdome (expiratório) e intercostal paraesternal (inspiratório). Para tanto, ratos da linhagem Wistar, divididos em grupos controle e treinado, foram submetidos à adaptação e, em seguida, ao treinamento da natação por $15,30,45$ e 60 dias, $1 \mathrm{~h} / \mathrm{dia}$, cinco dias/semana. Ao término do experimento os animais foram sacrificados, os músculos retirados e congelados em N-hexana resfriada a $-70^{\circ} \mathrm{C}$ em $\mathrm{N}_{2}$. Os cortes histológicos $(7 \mu \mathrm{m})$ foram feitos em micrótomo criostato $\mathrm{a}-20^{\circ} \mathrm{C}$, procedendo-se então à confecção das lâminas para a análise da morfologia geral (HE), análise da capacidade oxidativa (NADH-TR) e habilidade contrátil (ATPase miofibrilar), para verificação dos percentuais dos tipos de fibras musculares. Os músculos estudados revelaram fibras com contornos poligonais e com diferentes diâmetros, sendo de três tipos básicos; SO (slow oxydative), FOG (fast oxydative glycolitic) e FG (fast glycolitic), com distribuição em mosaico. A análise dos resultados demonstrou que o músculo reto do abdome apresentou aumento significativo nos percentuais de fibras SO e diminuição das fibras FOG nos animais que nadaram durante 60 dias, e aumento das fibras FG para os ratos que nadaram durante 15 dias. $\mathrm{O}$ músculo intercostal paraesternal apresentou aumento significativo nos percentuais das fibras SO

Recebido em 8/1/02

2- versão recebida em 6/5/02

Aceito em 7/7/02

Endereço para correspondência:

Paulo César Bálade Saad

Av. José Munia, 7.470, apto. 32-B, Jardim Vivendas

15090-500 - São José do Rio Preto, SP

Tel.: (17) 229-4996/(17) 9113-4158

E-mail: paulosaad@terra.com.br nos animais que nadaram durante 60 dias, aumento de fibras FOG para os que nadaram durante 45 e 60 dias e diminuição das fibras FG para os que nadaram durante 30,45 e 60 dias. Dos fatos, pode-se concluir que, no exercício da natação os músculos inspiratórios realizaram maior trabalho devido à pressão hidrostática, aumento da frequiência respiratória e aumento da resistência elástica pulmonar, e que, para isso, houve a necessidade de maior adaptação aeróbica, com conseqüente aumento do percentual de fibras oxidativas, induzindo maior resistência à fadiga.

Palavras-chave: Músculos respiratórios. Natação. Tipos de fibras musculares.

\section{ABSTRACT \\ Histologic and histochemical analysis of fibers of rectus of abdomen and paraesternal intercostal muscles of Wistar rats after swimming exercise}

The objective of the present study was to evaluate the influence of swimming exercise on metabolic and contractile abilities of rectus of abdomen and the paraesternal intercostal muscle fibers. Adult Wistar rats were initially submitted to an adaptation period and then divided into control and exercise groups. Exercised animals were submitted to swimming for 15, 30, 45, and 60 days, $1 \mathrm{hr} /$ day, 5days/ week. At the end of each period, the animals were killed. Small segments of the above muscles were frozen in $\mathrm{N}$-hexane at $-70^{\circ} \mathrm{C}$, pre-cooled with liquid nitrogen for two minutes. Histological transverse sections $(7 \mu \mathrm{m})$ were obtained in a cryostat microtome at $-20^{\circ} \mathrm{C}$. Some serial sections were stained with HE. Subsequent sections were reacted for $N A D H$-TR to evaluate the oxidizing capacity, and for m-ATPase, after acid and alkaline pre-incubations mediums. Under microscopic examination, the frequencies of fiber types were sampled. Muscle fibers revealed to be grouped in different fascicles, presenting polygonal outlines, with different diameters. Three kind of fibers were detected: SO (slow 
oxidative), FOG (fast oxidative glycolytic) and FG (fast glycolytic), presenting a mosaic pattern distribution. The analysis of the results demonstrated that after 60 days of exercise, the abdominal muscles showed a significant increase in the frequency of SO fibers. After 15 days, a significant decrease in frequency of intermediate FOG fibers and an increase of FG fibers. The paraesternal intercostal muscles revealed a significant increase in the percentage of so fibers in the 60 day-exercised animals, a percentage increase of FOG fibers in the animals of 45 and 60 days, and a percentage decrease of FG fibers in those of 30,45 and 60 days. So, due to hydrostatic pressure in swimming exercise, the degree of work was greater in the inspiration muscles. This increase was parallel with the increase of aerobic adaptation of fibers and fatigue resistance.

Key words: Respiratory muscles. Swimming exercise. Muscle fiber types.

\section{INTRODUÇÃO}

Tendo em vista serem várias as causas que provocam alterações das fibras musculares submetidas a exercícios regulares, procurou-se nesta pesquisa verificar se as alterações metabólicas e histológicas que, eventualmente, possam ocorrer em músculos submetidos ao exercício da natação permitem maior rendimento dos mesmos com melhor aproveitamento energético, o que, sem dúvida, explicaria a melhora observada no sistema respiratório de indivíduos treinados frente aos sedentários.

O treinamento de resistência é isoladamente o aspecto mais importante na reabilitação de pacientes com doença pulmonar crônica ${ }^{1} \mathrm{e}$ a atividade física da natação vem sendo utilizada como forma de aumentar a capacidade respiratória em indivíduos asmáticos, mostrando aumento da aptidão cardiorrespiratória e melhor adaptação aos exercí$\operatorname{cios}^{2}$. Os resultados vêm sendo avaliados através de provas de função pulmonar, gasometria arterial e questionários de qualidade de vida, mostrando melhora em jovens e ido$\operatorname{sos}^{3}$.

O aumento da resistência à fadiga dos músculos da ventilação pulmonar está entre os principais objetivos desse treinamento, justificando a melhora clínica dos indivíduos e sua melhor tolerância aos momentos de aumento do trabalho respiratório impostos por processos pulmonares obstrutivos e restritivos, característicos de tais estados fisiopatológicos ${ }^{4}$.

Dificuldades ventilatórias e intolerância aos exercícios são os distúrbios mais comuns na evolução dos pacientes com doenças pulmonares crônicas. A intolerância é multifatorial, mas, em doença mais grave, a limitação ventilatória é freqüentemente o fator limitante, alterando a mecâni- ca e função dos músculos respiratórios ${ }^{4}$. Com o aumento da ventilação durante o exercício, os indivíduos com limitação ao fluxo expiratório apresentam hiperinsuflação pulmonar dinâmica, aumentando o trabalho dos músculos inspiratórios e também proporcionando prejuízos hemodinâmicos pelo aumento da pressão intratorácica, com conseqüente diminuição da efetividade cardíaca ${ }^{4}$.

$\mathrm{O}$ aumento do fluxo de ar devido à elevada frequiência respiratória, juntamente com a pressão hidrostática exercida sobre o gradil costal durante o período de atividade da natação, seriam os fatores responsáveis para promover uma carga de trabalho imposta aos músculos ventilatórios, trazendo adaptações e conseqüente melhora na performance e resposta mecânica desta musculatura. As melhorias na qualidade de vida após o treinamento com exercícios são significantes, mas existem variações individuais imprevisíveis 5 .

Os músculos diafragma e intercostais têm sido estudados por vários autores em diversas situações de atividade física, demonstrando algumas controvérsias em seus resultados. Keens et al. ${ }^{6}$ relataram aumento da capacidade oxidativa de ambos os músculos através de cargas respiratórias aumentadas cronicamente (bandagem traqueal). Moore e Gollnick ${ }^{7}$, entretanto, verificaram aumento significativo apenas no músculo diafragma após 8-12 semanas de treinamento em esteira rolante, não havendo nenhuma mudança desta atividade nos músculos intercostais.

Controvérsias também existiram relacionadas aos músculos abdominais. Grinton et al. ${ }^{8}$ demonstraram não haver dados relativos aos efeitos do treinamento em tais músculos, contrastando com os inspiratórios principais (diafragma costal), em que já havia sido evidenciado aumento da capacidade oxidativa. Tal estudo demonstrou que o treinamento (esteira motorizada) resultou, entre outros dados, em elevada atividade de succinato desidrogenase (SDH) em dois músculos abdominais (reto e oblíquo externo), contrastando com os outros abdominais, que não apresentaram alteração significativa. Relataram ainda que, em geral, tais aumentos eram significativamente pequenos (aproximadamente 10 a 13\%) se comparados com o aumento no músculo plantar (aproximadamente 44\%), demonstrando, com esses dados, que o treinamento contínuo resultou em pequena mas, estatisticamente, significativa melhora na capacidade oxidativa dos músculos expiratórios. Uribe et al. ${ }^{9}$ observaram não haver nenhuma mudança significativa com o treinamento nos mesmos músculos, excluindo o reto do abdome, em que foi observado aumento de $26 \%$ de sua capacidade oxidativa. Segundo os autores, o aumento da atividade de SDH no músculo reto do abdome pode estar refletindo seu papel de apoio postural e/ou à locomoção, devido a nenhum outro músculo primário da expiração ter 
apresentado adaptação ao protocolo de treinamento. Verificaram, ainda, que os relativamente baixos níveis de atividade de SDH nos músculos abdominais sugeririam que eles não eram recrutados freqüentemente em repouso e que a falta de aumento com o treinamento, em ratos, indicava que esses músculos não contribuíam, significativamente, para a atividade ventilatória aumentada que acompanha os exercícios.

Reid et al. ${ }^{10}$ realizaram um estudo com o propósito de determinar o efeito de um programa de treinamento de resistência pela natação no tamanho e na percentagem dos tipos de fibras em diferentes regiões do músculo diafragma. Os resultados mostraram que as fibras do músculo na região torácica da face posterior eram menores nos animais do grupo de nadadores que no grupo controle, mas as percentagens dos tipos de fibras não eram diferentes entre os grupos, concluindo com isso que o treinamento pela natação poderia ter melhorado a resistência da região torácica posterior diminuindo a área de secção transversal e a distância para o oxigênio difundir-se às regiões internas das fibras do músculo.

Dessa forma, analisar as respostas quanto à percentagem dos tipos de fibras musculares após o exercício da natação nos músculos reto do abdome e intercostal paraesternal (um músculo expiratório e um inspiratório) poderia trazer dados importantes quanto às justificativas da melhora da atividade ventilatória pulmonar geral em indivíduos que praticam tal atividade.

Esta pesquisa teve por objetivo verificar, através de coloração histológica e reações histoquímicas, possíveis modificações quanto à capacidade oxidativa e ao percentual de fibras lentas (slow oxydative - SO) e rápidas (fast oxydative glycolitic - FOG e fast glycolitic - FG) nos músculos reto do abdome e intercostal paraesternal de ratos submetidos ao exercício físico da natação.

\section{MATERIAL E MÉTODOS}

Foram utilizados ratos Wistar (Rattus norvegicus), machos, adultos, com peso médio de 300 gramas. Os animais foram mantidos em gaiolas coletivas, com cinco animais, a $26^{\circ} \mathrm{C}$, e alimentados com ração padrão (Purina) e água ad libitum. A pesquisa foi realizada após a aprovação pelo Comitê de Ética da Universidade do Oeste Paulista - Unoeste, de acordo com as normas de manuseio e utilização de animais de laboratório (USA National Institutes of Health Guide for the Care and Use of Laboratory Animals).

Os animais foram divididos em grupo controle e grupo treinado:

Grupo controle: constituído por animais sem tratamento, sacrificados no início do experimento, denominados $\mathrm{C} 0$.
Grupo treinado: constituído por 20 animais, divididos em quatro subgrupos, submetidos à natação com duração de 60 minutos cada sessão, cinco dias por semana, perfazendo um total de três, seis, nove e 12 semanas de treinamento (15, 30, 45 e 60 dias), respectivamente, denominados subgrupos T1, T2, T3 e T4, sendo os mesmos sacrificados ao término do experimento, de acordo com o subgrupo em questão.

Com a finalidade de adaptá-los ao meio aquático, os animais foram submetidos a sessões de natação com duração de cinco, 15 e 30 minutos durante três dias. O treinamento foi realizado no período da manhã, em um tanque de vidro medindo $100 \times 50 \times 60 \mathrm{~cm}$, contendo água à temperatura de $30 \pm 1^{\circ} \mathrm{C}$.

A partir da segunda semana de treinamento, foi adicionado aos animais uma carga de 5\% do peso corporal (pesos de chumbo presos com elástico ao pescoço).

Após a última sessão de natação, de acordo com o grupo, os animais foram sacrificados através de injeção intraperitoneal de pentobarbital sódico ${ }^{11}$. Foram retirados os músculos reto do abdome e intercostal paraesternal para a análise histológica e histoquímica.

Os fragmentos dos músculos mediram $1,0 \mathrm{~cm}$ de comprimento $\mathrm{x} 0,5 \mathrm{~cm}$ de diâmetro. Os músculos foram congelados em $\mathrm{N}$-hexana, resfriada a $-70^{\circ} \mathrm{C}$ em $\mathrm{N}_{2}{ }^{12}$. Após tal procedimento, os blocos foram transferidos para a câmara do micrótomo criostato (Cryocut 1850 - Reichert-Young) e assim mantidos durante 20 a 30 minutos.

Os blocos foram fixados perpendicularmente aos suportes metálicos, usando-se para isso o adesivo (OCT - Tissue Tek Compound). A microtomia foi realizada a $-20^{\circ} \mathrm{C}$, com espessura de $7 \mu \mathrm{m}$.

Os cortes foram submetidos aos seguintes métodos:

a) Hematoxilina e eosina - HE, para avaliação das características morfológicas do tecido muscular ${ }^{13}$;

b) Nicotinamida adenina dinucleotídio tetrazólio reductase (NADH-TR), para avaliar a habilidade metabólica oxidativo-glicolítica dos tipos de fibras musculare ${ }^{14} \mathrm{e}$;

c) ATPase miofibrilar (mATPase), em pH 9,4, após incubação em meio alcalino (pH 10,4) e nos meios ácidos $(\mathrm{pH}$ 4,2; 4,4 e 4,6) para avaliar a habilidade contrátil rápida e lenta dos tipos de fibras musculares ${ }^{15}$.

A seguir, foram efetuadas a análise e a descrição da morfologia e das características de cada reação histoquímica das fibras musculares. Estas foram classificadas e nomeadas em SO, FOG e FG, conforme os critérios adotados por Petter et al. ${ }^{15}$, considerando, para tanto, seus diâmetros em cortes transversais, a intensidade e distribuição citológica do produto da reação para metabolismo oxidativo e glicolítico à reação NADH-TR, e a reatividade à reação para ATPase miofibrilar, após pré-incubação em meio alcalino e nos 

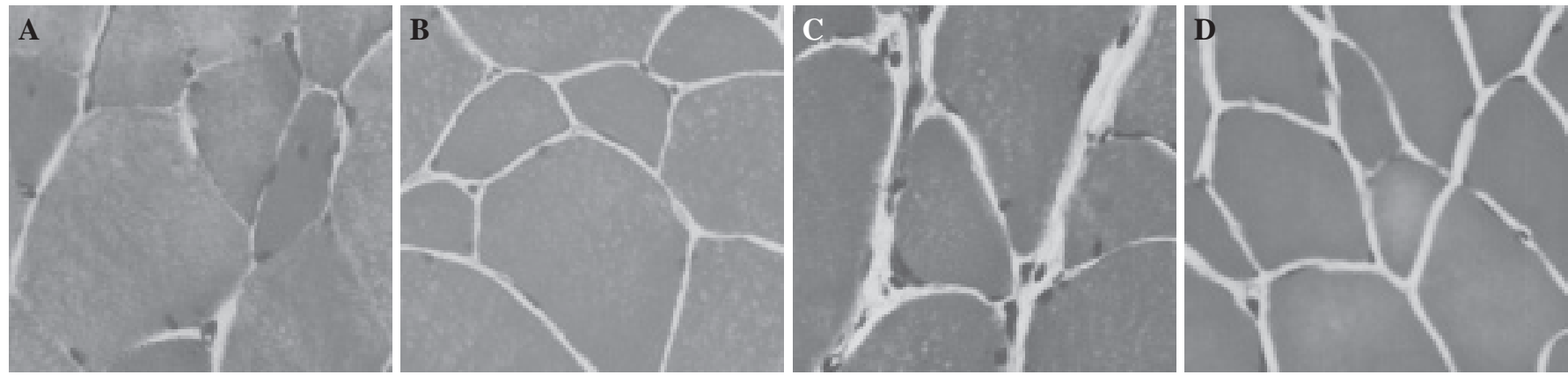

Fig. 1 - Cortes transversais dos músculos reto do abdome e intercostal paraesternal (HE, 400x). A) músculo reto do abdome - grupo controle (C0); B) músculo reto do abdome - grupo treinado (T4); C) músculo intercostal paraesternal - grupo controle (C0); D) músculo intercostal paraesternal - grupo treinado (T4).
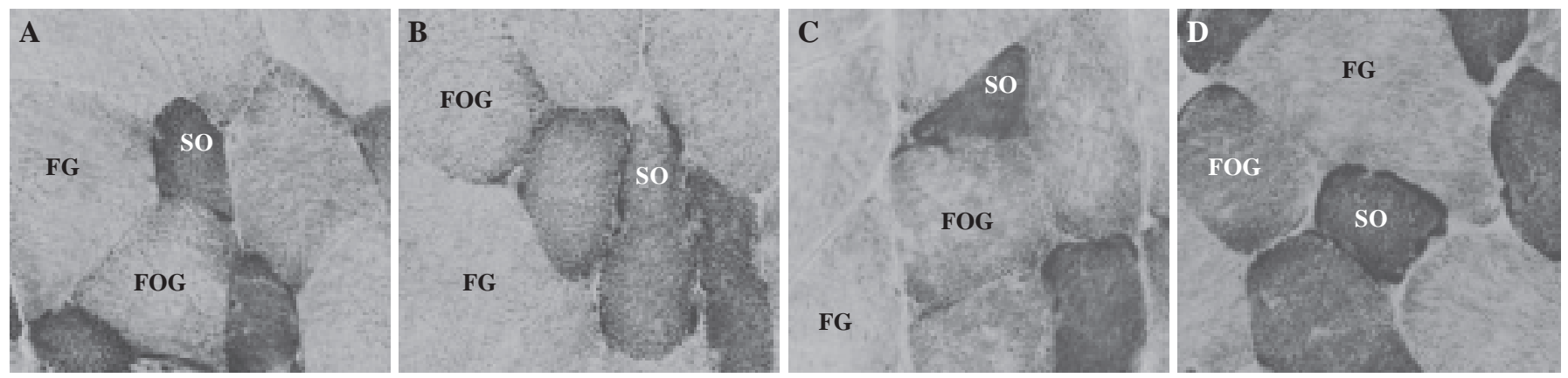

Fig. 2 - Cortes transversais dos músculos reto do abdome e intercostal paraesternal (NADH-TR, 400x). Fibras aeróbicas (SO e FOG); fibras anaeróbicas $(\boldsymbol{F G})$. A) músculo reto do abdome - grupo controle (C0); B) músculo reto do abdome - grupo treinado (T4); $\boldsymbol{C}$ ) músculo intercostal paraesternal - grupo controle (CO); D) músculo intercostal paraesternal - grupo treinado (T4).

meios ácidos, para avaliar a habilidade contrátil dos tipos de fibras musculares.

Utilizando-se um microscópio óptico, foram amostrados as freqüências (\%) dos tipos de fibras, em amostras de no mínimo 200 fibras. Na reação NADH-TR, as fibras escuras representavam as fibras oxidativas (SO e FOG) e as claras, as fibras glicolíticas (FG); na reação mATPase ácida (pH4,4), as escuras representavam as fibras de contração lenta (SO) e as claras, as fibras de contração rápida (FOG e FG). O inverso, escuras para as de contração rápida e claras para as de contração lenta, na mATPase alcalina (pH 10,4).

Os dados foram submetidos à analise estatística, por delineamento inteiramente casualisado. Quando, na análise de variância, houve diferença significante entre as médias, utilizou-se o teste de Tukey para se fazer as comparações pareadas de duas médias ${ }^{16}$. Diferenças foram consideradas significantes quando valor $\mathrm{p}<\alpha=0,05$.

\section{RESULTADOS}

Os cortes corados pela HE revelaram que as fibras dos dois músculos estudados possuem diferentes diâmetros, contornos poligonais e vários núcleos em posição periféri- ca ao citoplasma, sem qualquer diferencial entre os animais dos dois grupos experimentais (fig. 1).

A tipagem das fibras foi determinada pela análise histoquímica através das reações mATPase e NADH-TR (figs. 2, 3 e 4).

A tabela 1 apresenta os valores médios (em percentagem) das fibras (SO, FOG e FG) do músculo reto do abdome dos animais dos grupos controle (C0) e dos subgrupos T1,

\begin{tabular}{|c|c|c|c|c|c|c|c|}
\hline & \multicolumn{6}{|c|}{$\begin{array}{c}\text { TABELA } 1 \\
\text { Valores médios (em percentagem) das fibras } \\
\text { SO, FOG e FG do músculo reto do abdome } \\
\text { dos ratos dos grupos experimentais }\end{array}$} & \multirow[b]{2}{*}{ DP } \\
\hline & $\mathbf{n}$ & so & DP & FOG & DP & FG & \\
\hline $\mathrm{CO}$ & 5 & $15,56 \mathrm{~b}$ & 3,25 & $32,59 \mathrm{a}$ & 7,98 & $51,84 \mathrm{~b}$ & 5,03 \\
\hline $\mathrm{T} 1$ & 5 & $23,50 a b$ & 6,46 & $13,85 \mathrm{~b}$ & 6,32 & 62,65 a & 0,87 \\
\hline $\mathrm{T} 2$ & 5 & $23,15 a b$ & 8,12 & $24,57 \mathrm{ab}$ & 9,93 & $52,27 \mathrm{~b}$ & 3,47 \\
\hline T3 & 5 & $21,66 a b$ & 5,52 & $25,13 a b$ & 9,63 & $53,20 \mathrm{~b}$ & 6,30 \\
\hline $\mathrm{T} 4$ & 5 & $28,83 a$ & 6,57 & $19,61 \mathrm{ab}$ & 1,92 & $51,55 \mathrm{~b}$ & 6,28 \\
\hline
\end{tabular}



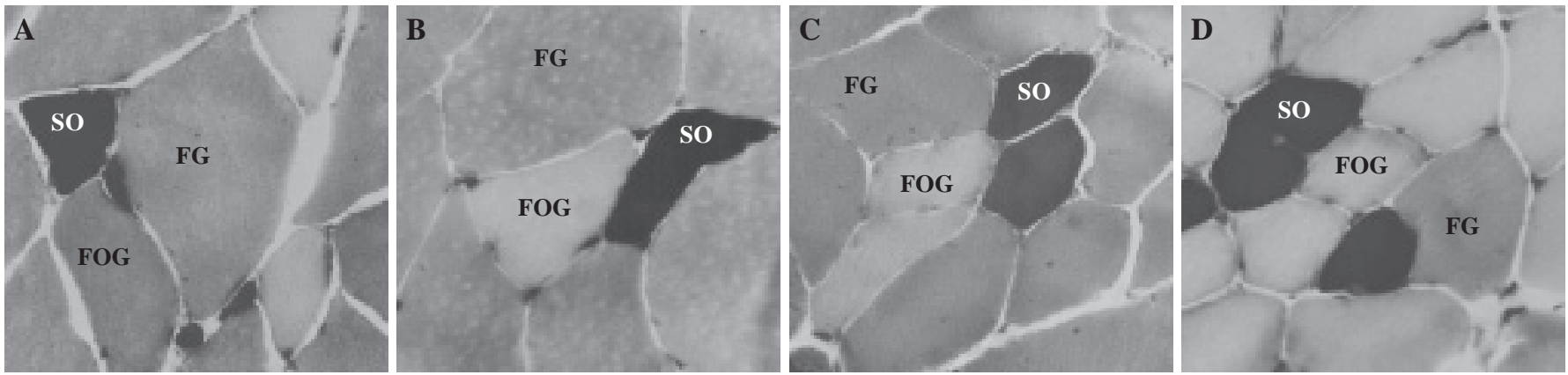

Fig. 3 - Cortes transversais dos músculos reto do abdome e intercostal paraesternal. mATPase (pH 4,4), 400x. Fibras lentas (SO); fibras rápidas (FOG e $\boldsymbol{F G ) . ~ A ) ~ m u ́ s c u l o ~ r e t o ~ d o ~ a b d o m e ~ - ~ g r u p o ~ c o n t r o l e ~ ( C O ) ; ~ B ) ~ m u ́ s c u l o ~ r e t o ~ d o ~ a b d o m e ~ - ~ g r u p o ~ t r e i n a d o ~ ( T 4 ) ; ~} \boldsymbol{C})$ músculo intercostal paraesternal - grupo controle (CO); D) músculo intercostal paraesternal - grupo treinado (T4).
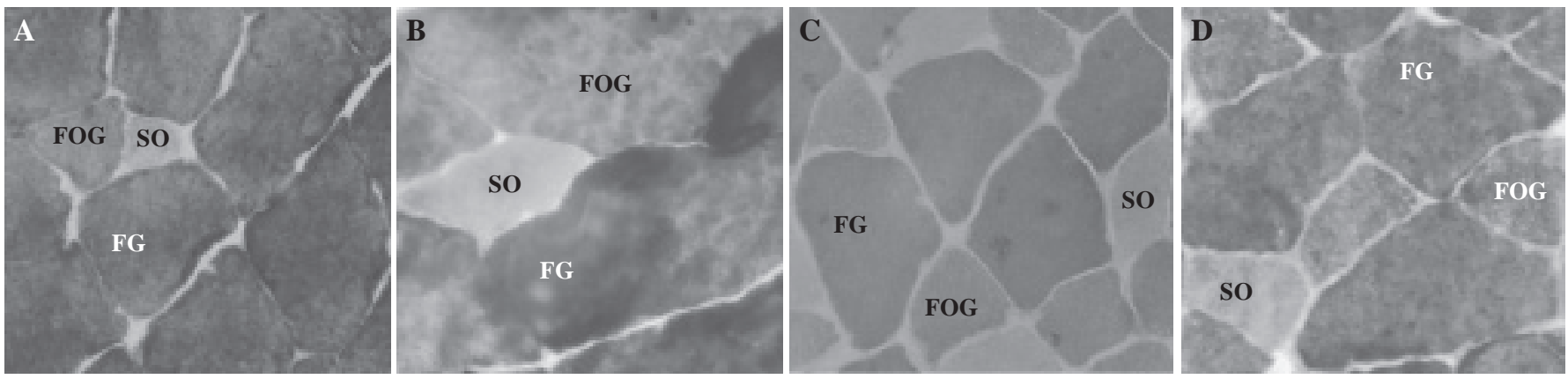

Fig. 4 - Cortes transversais dos músculos reto do abdome e intercostal paraesternal. mATPase (pH 10,4), 400x. Fibras lentas (SO); fibras rápidas (FOG e $\boldsymbol{F G ) . ~ A ) ~ m u ́ s c u l o ~ r e t o ~ d o ~ a b d o m e ~ - ~ g r u p o ~ c o n t r o l e ~ ( C O ) ; ~ B ) ~ m u ́ s c u l o ~ r e t o ~ d o ~ a b d o m e ~ - ~ g r u p o ~ t r e i n a d o ~ ( T 4 ) ; ~} \boldsymbol{C})$ músculo intercostal paraesternal - grupo controle (CO); D) músculo intercostal paraesternal - grupo treinado (T4).

T2, T3 e T4 (15, 30, 45 e 60 dias de natação, respectivamente).

Os animais treinados apresentaram maior percentual de fibras lentas oxidativas (SO) quando comparados com os do grupo controle. Através da análise de variância e do teste de Tukey, pode-se observar que os valores são estatisticamente significativos $(\mathrm{p}<0,05)$ apenas entre os animais controle e os do subgrupo T4 (60 dias). Todavia, pode ser também verificado não haver diferenças entre os subgrupos de animais treinados.

Os valores percentuais do número de fibras rápidas oxidativas glicolíticas (FOG) de todos os animais treinados apresentaram menor percentual quando comparados com os do grupo controle, sendo os valores estatisticamente significativos $(p<0,05)$ apenas entre os animais controle e os do subgrupo T1 (15 dias), não havendo diferenças entre os subgrupos de animais treinados.

Pode-se observar também que os animais dos subgrupos T1, T2 e T3 apresentaram maior percentual de fibras rápidas glicolíticas (FG) e os do subgrupo T4 mostraram pequena diminuição dessas fibras, quando comparados com os do grupo controle, sendo, porém, esses valores estatistica-

\section{8}

mente significativos $(\mathrm{p}<0,05)$ apenas para o grupo T1, não havendo diferenças significativas entre os demais grupos.

A tabela 2 apresenta os valores médios (em percentagem) das fibras (SO, FOG e FG) do músculo intercostal paraesternal dos animais dos grupos controle $(\mathrm{C} 0)$ e dos subgrupos T1, T2, T3 e T4 (15, 30, 45 e 60 dias de natação, respectivamente).

\section{TABELA 2}

Valores médios (em percentagem) das fibras SO, FOG e FG do músculo intercostal paraesternal dos ratos dos grupos experimentais

\begin{tabular}{lccccccc}
\hline & $\mathbf{n}$ & SO & DP & FOG & DP & FG & DP \\
& & & & & & & \\
CO & 5 & $22,86 \mathrm{~b}$ & 4,72 & $33,33 \mathrm{~b}$ & 9,27 & $43,8 \mathrm{a}$ & 7,10 \\
T1 & 5 & $21,16 \mathrm{~b}$ & 4,48 & $34,96 \mathrm{~b}$ & 4,40 & $43,88 \mathrm{a}$ & 6,17 \\
T2 & 5 & $26,34 \mathrm{ab}$ & 3,50 & $43,04 \mathrm{ab}$ & 7,08 & $30,61 \mathrm{~b}$ & 5,18 \\
T3 & 5 & $25,7 \mathrm{ab}$ & 4,22 & $53,23 \mathrm{a}$ & 5,11 & $21,06 \mathrm{~b}$ & 6,47 \\
T4 & 5 & $31,36 \mathrm{a}$ & 2,74 & $47,81 \mathrm{a}$ & 6,62 & $20,82 \mathrm{~b}$ & 4,97 \\
\hline
\end{tabular}

* Letras diferentes indicam diferença estatisticamente significante $(p<0,05)$. 
Pode-se observar que os animais dos subgrupos T2, T3 e T4 apresentaram maior percentual de fibras lentas oxidativas (SO) e os do subgrupo T1, pequena diminuição desse percentual, quando comparados com os animais do grupo controle, mostrando-se estatisticamente significativos ( $\mathrm{p}<$ $0,05)$ para o grupo $\mathrm{T} 4$ frente ao grupo controle e subgrupo T1.

Os animais treinados apresentaram maior percentual de fibras rápidas oxidativas glicolíticas (FOG) quando comparados com os animais do grupo controle, estatisticamente significativo $(\mathrm{p}<0,05)$ para os grupo T3 e T4 frente ao grupo controle e subgrupo T1.

Pode-se ainda observar que os animais dos subgrupos $\mathrm{T} 2$, T3 e T4 apresentaram menor percentual de fibras rápidas glicolíticas (FG) e os do grupo T1, pequeno aumento dessas fibras, quando comparados com os animais do grupo controle, sendo os valores estatisticamente significativos $(\mathrm{p}<0,05)$ para os grupo T2, T3 e T4 frente ao grupo controle e subgrupo T1.

Com relação à capacidade oxidativa, a tabela 3 mostra os valores percentuais das fibras que apresentaram reação positiva ao NADH-TR (oxidativas) dos músculos reto do abdome e intercostal paraesternal dos ratos dos grupos controle $(\mathrm{C} 0)$ e treinado $(\mathrm{T} 1, \mathrm{~T} 2, \mathrm{~T} 3 \mathrm{e} \mathrm{T} 4)$.

\section{TABELA 3}

Valores médios (em percentagem) das fibras que apresentaram reação positiva ao NADH-TR (oxidativas) dos músculos reto do abdome e intercostal paraesternal dos ratos dos grupos experimentais
Reto do abdome

$$
\begin{aligned}
& 48,15 a \\
& 37,35 \mathrm{~b} \\
& 47,47 \mathrm{a} \\
& 46,79 \mathrm{a} \\
& 48,44 \mathrm{a}
\end{aligned}
$$

Intercostal paraesternal

$56,19 b$

$56,12 \mathrm{~b}$

69,38 a

78,94 a

79,18 a
* Letras diferentes indicam diferença estatisticamente significante $(p<0,05)$.

Pode-se observar que, no músculo reto do abdome, os animais do subgrupo T4 apresentaram maior percentual de fibras oxidativas e os dos subgrupos T1, T2 e T3, menor percentual, quando comparados com os animais do grupo controle, sendo estatisticamente significativos $(\mathrm{p}<0,05)$ para o subgrupo T1 frente ao grupo controle e aos demais subgrupos.

Com relação ao músculo intercostal paraesternal, os animais do subgrupo T1 apresentaram menor percentual de fibras oxidativas e os dos subgrupos T2, T3 e T4, maior percentual em ordem crescente, quando comparados com os animais do grupo controle, mostrando serem os valores estatisticamente significativos $(\mathrm{p}<0,05)$ para os subgrupos T2, T3 e T4 frente ao grupo controle e ao subgrupo T1 .

\section{DISCUSSÃO}

Os resultados observados sugerem que o exercício da natação possa apresentar adaptações no músculo reto do abdome, promovendo aumento percentual de fibras lentas, o que pode ser de grande importância na manutenção da ventilação pulmonar, mantendo maior fluxo de ar na expiração, promovendo maior freqüência respiratória, com conseqüente aumento do volume minuto ventilatório e, para isso, há necessidade de fibras com grande resistência à fadiga, justificando a melhor performance dos indivíduos treinados na manutenção de atividades continuadas, a despeito do verificado por Foss e Keteyian ${ }^{17}$ quando relataram que a distribuição das fibras lentas e rápidas não pode ser alterada em qualquer grau significativo pelo treinamento.

Outro aspecto a ser observado é que o aumento de fibras FG e a diminuição de fibras FOG no músculo reto do abdome dos animais do subgrupo T1 parecem estar relacionados à grande carga de trabalho na manutenção da postura durante a fase inicial do treinamento, igualmente relatado por Uribe et al. ${ }^{9}$, parecendo ser gradativa a adaptação dos animais. Isso pode ser observado em melhor aceitação e desenvoltura dos mesmos após esse período, quando então começaram a aumentar novamente a percentagem do número de fibras oxidativas, retornando aos valores do grupo controle.

Os resultados observados no músculo intercostal paraesternal demonstraram aumento significativo da capacidade oxidativa nos animais dos subgrupos T2, T3 e T4 (percentual de fibras SO e FOG) e de fibras lentas oxidativas (SO) apenas nos ratos que nadaram por 60 dias (subgrupo T4), quando comparados com o subgrupo T1 e o grupo controle (C0), a despeito do que relataram Keens et al. ${ }^{6}$, que observaram aumento da capacidade oxidativa dos músculos diafragma e intercostal, porém aumento de fibras lentas apenas no diafragma, e o descrito por Moore e Gollnick $^{7}$, que não encontraram mudança da capacidade oxidativa nos músculos intercostais.

As fibras rápidas glicolíticas (FG) do músculo intercostal paraesternal apresentaram-se diminuídas significativamente nos ratos que nadaram durante 30,45 e 60 dias (subgrupos $\mathrm{T} 2, \mathrm{~T} 3 \mathrm{e} \mathrm{T} 4$, respectivamente), quando comparadas as fibras dos ratos do grupo controle $(\mathrm{C} 0)$ e dos que nadaram durante 15 dias (subgrupo T1), demonstrando a transformação relatada por McArdle et al..$^{18}$ e negada por Foss e Keteyian ${ }^{17}$. O valor percentual para as fibras FG do músculo intercostal paraesternal a partir de 30 dias de natação apresentou relação inversamente proporcional à duração 
da atividade, ou seja, quanto maior o número de dias de atividade, menor o valor percentual das mesmas, sendo tal decréscimo coincidente com o aumento das fibras SO e FOG, confirmando suas modificações.

Os músculos inspiratórios durante seu trabalho normal têm que vencer resistências elásticas, inelásticas e de fricção, dentre outras, com o intuito de promover a entrada do ar nas vias aéreas. Quando de uma atividade física, algumas dessas resistências tornam-se aumentadas como, por exemplo, quando, devido ao aumento da freqüência, ocorre aumento do fluxo com consequiente turbilhonamento do ar. Além disso, a hiperinsuflação provoca aumento da resistência elástica devido à diminuição da complacência dinâmica pulmonar. Aliado a esses fatores, deve-se levar em consideração que o menor tempo expiratório implica maior relação do tempo inspiratório com o tempo total da ventilação.

Pode-se concluir que todos esses fatos somados fazem com que os músculos inspiratórios tenham que realizar maior trabalho e que para isso haveria a necessidade de maior adaptação aeróbica, com conseqüente aumento do

\section{REFERÊNCIAS}

1. Cooper CB. Exercise in chronic pulmonary disease: aerobic exercise prescription. Med Sci Sports Exerc 2001;33:S671-9.

2. Varray A, Mercier J, Terral C, Prefaut C. Effects of individualized aerobic training in the readaptation of the asthmatic child to exercise. Rev Mal Respir 1990;7:581-7.

3. Kurabayashi H, Kubota K, Machida I, Tamura K, Take H, Shirakura T. Effective physical therapy for chronic obstructive pulmonary disease. Pilot study of exercise in hot spring water. Am J Phys Med Rehabil 1997; 76:204-7.

4. O'Donnell DE. Ventilatory limitations in chronic obstructive pulmonary disease. Med Sci Sports Exerc 2001;33:S647-55.

5. Troosters T, Gosselink R, Decramer M. Exercise training in COPD: how to distinguish responders from nonresponders. J Cardiopulm Rehabil 2001;21:10-7.

6. Keens TG, Chen V, Patel P, O'Brien P, Levison H, Ianuzzo CD. Cellular adaptations of the ventilatory muscles to a chronic increased respiratory load. J Appl Physiol 1978;44:905-8.

7. Moore RL, Gollnick PD. Response of ventilatory muscles of the rat to endurance training. Pflugers Arch 1982;392:268-71.

8. Grinton S, Powers SK, Lawler J, Criswell D, Dodd S, Edwards W. Endurance training-induced increases in expiratory muscle oxidative capacity. Med Sci Sports Exerc 1992;24:551-5.

9. Uribe JM, Stump CS, Tipton CM, Fregosi RF. Influence of exercise training on the oxidative capacity of rat abdominal muscle. Respir Physiol 1992;88:171-80. percentual do número de fibras oxidativas, induzindo maior resistência à fadiga. Deve-se levar em consideração, também, que nesta pesquisa os animais praticaram o exercício da natação, o que acarretou uma pressão hidrostática sobre o tórax que dificultava ainda mais o trabalho inspiratório.

Todavia, deve-se ressaltar que essa pressão hidrostática e a resistência elástica aumentada são fatores que favorecem o mecanismo expiratório, parecendo demonstrar que, durante a atividade da natação, há maior carga de trabalho aos músculos inspiratórios comparados com os expiratórios, o que pode ser verificado também nesta pesquisa.

Para melhor elucidação do que foi observado, há necessidade de novas pesquisas sobre fibras musculares de outros músculos da respiração, como, por exemplo, os outros abdominais, os intercostais internos, diafragma e os demais envolvidos com a ventilação pulmonar.

Todos os autores declararam não haver qualquer potencial conflito de interesses referente a este artigo.

10. Reid WD, Shanks J, Samrai B. Regional and fiber-type percentages and sizes in the hamster diaphragm after swim training. Phys Ther 1997;77: $178-86$.

11. Marshall S, Milligan A, Yates R. Experimental techniques and anaesthesia in the rat and mouse. Anzccarte. Facts Sheet, Anzccart News, 1994; $7: 4$.

12. Dal Pai V. Histoenzimologia: teoria e prática. Instituto de Biociências Unesp, Botucatu, 1995.

13. McManus JFA, Mowry RW. Staining methods: histologic and histochemical medical division. New York: Harper \& Brother, 1960;283-310.

14. Dubowitz V, Brooke MH, Neville H. Muscle biopsy: a modern approach. London: Saunders Co. Ltd., 1972;471.

15. Peter JB, Barnard RJ, Edgerton VR, Gillespie CA, Stempel KE. Metabolic profiles of three fiber types of skeletal muscle in guinea pigs and rabbits. Biochemistry 1972;11:2627-37.

16. Berquo ES, Souza JMP, Gotlieb SLD, Souza JFP. Bioestatística. São Paulo: Edusp, 1981;350.

17. Foss ML, Keteyian SJ. Fox - Bases fisiológicas do exercício e do espor-

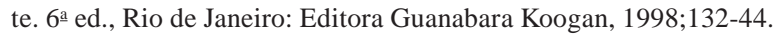

18. McArdle WD, Katch FI, Katch VL. Fisiologia do exercício - energia, nutrição e desempenho humano. 4a ed., Rio de Janeiro: Editora Guanabara Koogan, 1998;314-9. 